\title{
Estimation of Radar Cross Section of a Target under Track
}

\author{
Young-Hun Jung, ${ }^{1}$ Sun-Mog Hong, ${ }^{2}$ and Seung Ho Choi ${ }^{3}$ \\ ${ }^{1}$ Agency for Defense Development, Yuseong P.O. Box 35-1, Daejeon 305-600, Republic of Korea \\ ${ }^{2}$ School of EE, Kyungpook National University, Daegu 702-701, Republic of Korea \\ ${ }^{3}$ Department of EIE, Seoul National University of Technology, Seoul 139-743, Republic of Korea \\ Correspondence should be addressed to Sun-Mog Hong, smhong@ee.knu.ac.kr \\ Received 19 April 2010; Accepted 6 October 2010 \\ Academic Editor: Frank Ehlers
}

Copyright (๑) 2010 Young-Hun Jung et al. This is an open access article distributed under the Creative Commons Attribution License, which permits unrestricted use, distribution, and reproduction in any medium, provided the original work is properly cited.

In allocating radar beam for tracking a target, it is attempted to maintain the signal-to-noise ratio (SNR) of signal returning from the illuminated target close to an optimum value for efficient track updates. An estimate of the average radar cross section (RCS) of the target is required in order to adjust transmitted power based on the estimate such that a desired SNR can be realized. In this paper, a maximum-likelihood (ML) approach is presented for estimating the average RCS, and a numerical solution to the approach is proposed based on a generalized expectation maximization (GEM) algorithm. Estimation accuracy of the approach is compared to that of a previously reported procedure.

\section{Introduction}

Beam allocation in phased array radar tracking is a wellknown problem, and it has been addressed in a large body of literature [1-6] and the references therein. A goal of the allocation is to minimize the use of radar resources while maintaining a target under track. In the allocation for a track update, one of the principal parameters to be adjusted is transmitted power. The transmitted power has an impact on the signal-to-noise ratio (SNR) of return signal from an illuminated target. The SNR is directly proportional to the transmitted power and the radar cross section (RCS) of the target $[7,8]$.

It is often attempted to maintain SNR close to an optimum value for efficient track updates. The transmitted power is adjusted in the attempt such that a desired SNR can be realized. Since the target RCS is unknown, it is required to adjust the power based on an estimate of the average RCS [7]. An algorithmic procedure for estimating the average RCS was proposed in $[1,2]$. The procedure, denoted by MED, adjusts the RCS estimate, depending on the median difference between the SNR measurements of return signals in a sliding window and their corresponding expected values. In this paper, a maximum-likelihood (ML) approach is presented for estimating the average RCS, and a numerical solution to the approach is proposed based on a generalized expectation maximization (GEM) algorithm. Numerical experiments were performed to compare estimation performance of the ML approach with that of MED. The experimental results show that ML estimation can perform successfully even for a low SNR target that MED fails to estimate.

\section{RCS Model}

The radar cross section depends on many factors, including electromagnetic scattering properties of a target and aspect angles, and it is often statistically characterized by a Swerling model [7]. We assume that the fluctuation model of target RCS under consideration is Swerling I. The received signal strength of a target with the fluctuation varies independently from scan to scan, and it is characterized as an exponential random variable. The signal strength at scan $k$ normalized with respect to the noise spectral density, denoted by $z_{k}$, has a probability density function (pdf)

$$
f\left(z_{k}\right)=\frac{1}{1+\overline{\mathrm{SNR}}_{k}} \cdot \exp \left(-\frac{z_{k}}{1+\overline{\mathrm{SNR}}_{k}}\right),
$$

where $\overline{\mathrm{SNR}}_{k}$ denotes the average SNR at scan $k$. Note that $\overline{\mathrm{SNR}}_{k}$ is proportional to the average RCS, denoted by $\bar{\sigma}$. That 
is, $\overline{\mathrm{SNR}}_{k}=\alpha_{k} \bar{\sigma}$, where $\alpha_{k}$ is a known constant that depends on target range and transmitted power at $k$ [7].

The detection of a target takes place when the received signal strength is higher than a specified threshold that can be represented in terms of the false alarm probability $P_{F}$. To be specific, the detection occurs if

$$
z_{k} \geq-\ln P_{F}
$$

The target detection of (2) defines the relationship between the probability of detection at $k\left(P_{D k}\right)$, the false alarm probability $P_{F}$, and $\overline{\mathrm{SNR}}_{k}$ such that

$$
P_{D k}=P_{F}^{1 /\left(1+\overline{\mathrm{SNR}}_{k}\right)} .
$$

The false alarm probability $P_{F}$ represents the probability of detecting a false measurement due to noise interference. Note that $P_{F}$ is a predetermined constant.

\section{ML Estimation of RCS}

An algorithmic procedure for estimating the average RCS was proposed in $[1,2]$. The procedure (denoted by MED) adjusts the average RCS estimate by $0.5 \mathrm{~dB}$ whenever the median difference between SNR measurements of return signals in a sliding window and their corresponding expected values is $1 \mathrm{~dB}$ or greater. In the case of a missed detection, the estimate is decreased by $0.5 \mathrm{~dB}$. We adopt the acronym MED to represent the procedure, since it uses the median as its statistic. In this section, a maximum-likelihood approach for estimating the average RCS $\bar{\sigma}$ and its numerical solution is presented. Along the context of the problem posed in $[1,2]$, we assume that the false alarm probability $P_{F}$ is small enough so that, if a detection occurs, it is from the target under track. Under this assumption, we obtain an estimate of $\bar{\sigma}$ from a sequence of detections and misses over a sliding window.

Suppose that $\bar{\sigma}$ is constant over a sliding window, and suppose that we have a sequence of $N$ detections and $M$ missed detections in the window. Denote by $D$ the index set of scans with detection and by $\bar{D}$ the index set of scans with no detection. Also, let us denote by $\mathscr{D}_{k}$ the event that detection occurs at scan $k$. Since the received signal strength is independent from scan to scan, the misses and detections form an independent sequence. Specifically, the probability-pdf of the observations is given by $c \cdot\left(\prod_{i \in D} f\left(z_{i}\right)\right.$ $\left.\left.\mathscr{D}_{i}\right)\right) \cdot\left(\prod_{i \in D} P\left[\mathscr{D}_{i}\right]\right) \cdot\left(\prod_{j \in \bar{D}}\left(1-P\left[\mathscr{D}_{j}\right]\right)\right)$, where $c$ is the normalizing constant and $f\left(z_{i} \mid \mathscr{D}_{i}\right)$ is the conditional pdf of signal strength given the event $\mathscr{D}_{i}$. Substituting (1) and (3) with $\overline{\mathrm{SNR}}_{k}=\alpha_{k} \bar{\sigma}$ into the probability-pdf, we obtain the incomplete-data log-likelihood function for the parameter $\bar{\sigma}$, which is given by

$$
\begin{aligned}
\ln L(\bar{\sigma})= & -\sum_{i \in D}\left(\ln \left(1+\alpha_{i} \bar{\sigma}\right)+\frac{z_{i}}{1+\alpha_{i} \bar{\sigma}}\right) \\
& +\sum_{j \in \bar{D}} \ln \left(1-P_{F}^{1 /\left(1+\alpha_{j} \bar{\sigma}\right)}\right) .
\end{aligned}
$$

The maximum-likelihood estimate of the average RCS is represented by

$$
\bar{\sigma}_{M L}=\arg \max _{\bar{\sigma}} \ln L(\bar{\sigma}) .
$$

The ML solution to (5) appears to be analytically intractable, and we apply the expectation maximization (EM) algorithm to obtain the solution. The EM algorithm is an efficient iterative procedure for finding the ML estimate of model parameters from a given data set in the presence of incomplete or missing data [9]. Note that various targettracking problems have been formulated and solved in the framework of the EM algorithm [10-15]. Let us denote by $\bar{D}_{j}$ the event that a miss occurs at scan $j$, and by $y_{j}$ the unknown (or missing) signal strength of the miss. The complete-data likelihood function $L_{c}(\bar{\sigma})$ for the parameter $\bar{\sigma}$ is defined by

$$
\begin{aligned}
L_{c}(\bar{\sigma})= & \left(\prod_{i \in D} f\left(z_{i} \mid \mathscr{D}_{i}\right)\right) \cdot\left(\prod_{i \in D} P\left[\mathscr{D}_{i}\right]\right) \\
& \cdot\left(\prod_{j \in \bar{D}} P\left[\overline{\mathscr{D}}_{j}\right]\right) \cdot\left(\prod_{j \in \bar{D}} f\left(y_{j} \mid \overline{\mathscr{D}}_{j}\right)\right),
\end{aligned}
$$

where $f\left(y_{j} \mid \overline{\mathscr{D}}_{j}\right)$ is the conditional pdf of the missing signal strength given the event $\overline{\mathcal{D}}_{j}$, that is,

$$
\begin{array}{r}
f\left(y_{j} \mid \bar{D}_{j}\right)=\left(1-P_{F}^{1 /\left(1+\alpha_{j} \bar{\sigma}\right)}\right)^{-1} \frac{1}{1+\alpha_{j} \bar{\sigma}} \exp \left(-\frac{y_{j}}{1+\alpha_{j} \bar{\sigma}}\right), \\
0<y_{j}<-\ln P_{F},
\end{array}
$$

and zero, otherwise. The complete-data likelihood function can be written as

$$
\begin{aligned}
L_{c}(\bar{\sigma})= & \prod_{i \in D} \frac{1}{1+\alpha_{i} \bar{\sigma}} \exp \left(-\frac{z_{i}}{1+\alpha_{i} \bar{\sigma}}\right) \\
& \cdot \prod_{j \in \bar{D}} \frac{1}{1+\alpha_{j} \bar{\sigma}} \exp \left(-\frac{y_{j}}{1+\alpha_{j} \bar{\sigma}}\right),
\end{aligned}
$$

and the complete-data log-likelihood function is given by

$$
\begin{aligned}
\ln L_{c}(\bar{\sigma})= & -\sum_{i \in D}\left(\ln \left(1+\alpha_{i} \bar{\sigma}\right)+\frac{z_{i}}{1+\alpha_{i} \bar{\sigma}}\right) \\
& -\sum_{j \in \bar{D}}\left(\ln \left(1+\alpha_{j} \bar{\sigma}\right)+\frac{y_{j}}{1+\alpha_{j} \bar{\sigma}}\right) .
\end{aligned}
$$

Define by $Q\left(\bar{\sigma}, \bar{\sigma}^{(l-1)}\right)$ the expectation of the complete-data log-likelihood function with respect to the unknown signal strength, given the observations $\left\{z_{i}: i \in D\right\}$ and the current parameter estimate $\bar{\sigma}^{(l-1)}$. That is,

$$
Q\left(\bar{\sigma}, \bar{\sigma}^{(l-1)}\right)=E\left[\ln L_{c}(\bar{\sigma}) \mid\left\{z_{i}: i \in D\right\}, \bar{\sigma}^{(l-1)}\right] .
$$


In the $l$ th iteration of the EM algorithm, the expectation $Q\left(\bar{\sigma}, \bar{\sigma}^{(l-1)}\right)$ is maximized with respect to $\bar{\sigma}$, and $\bar{\sigma}^{(l)}$ is updated with the maximizer as

$$
\bar{\sigma}^{(l)}=\arg \max _{\bar{\sigma}} Q\left(\bar{\sigma}, \bar{\sigma}^{(l-1)}\right) .
$$

Note that each iteration is guaranteed to increase the log-likelihood function (4) and the EM algorithm leads $\bar{\sigma}^{(l)}$ ultimately to the value that maximizes the likelihood function [9].

The conditional expectation $Q\left(\bar{\sigma}, \bar{\sigma}^{(l-1)}\right)$ of the completedata log-likelihood function (9) can be rewritten as

$$
\begin{aligned}
Q\left(\bar{\sigma}, \bar{\sigma}^{(l-1)}\right)= & -\sum_{i \in D}\left(\ln \left(1+\alpha_{i} \bar{\sigma}\right)+\frac{z_{i}}{1+\alpha_{i} \bar{\sigma}}\right) \\
& -\sum_{j \in \bar{D}}\left(\ln \left(1+\alpha_{j} \bar{\sigma}\right)+\frac{g\left(\alpha_{j} \bar{\sigma}^{(l-1)}\right)}{1+\alpha_{j} \bar{\sigma}}\right),
\end{aligned}
$$

where $g\left(\alpha_{j} \bar{\sigma}^{(l-1)}\right)=E\left[y_{j} \mid \bar{\sigma}^{(l-1)}\right]$ that is given by

$$
\begin{aligned}
g\left(\alpha_{j} \bar{\sigma}^{(l-1)}\right)= & \left(1+\alpha_{j} \bar{\sigma}^{(l-1)}\right) \\
& +\left(1-P_{F}^{1 /\left(1+\alpha_{j} \bar{\sigma}^{(l-1)}\right)}\right)^{-1} P_{F}^{1 /\left(1+\alpha_{j} \bar{\sigma}^{(l-1)}\right)} \ln P_{F} .
\end{aligned}
$$

Unfortunately, it appears infeasible to obtain the maximizer of (12) in an analytic form. Instead, we evaluate $\bar{\sigma}^{(l)}$ in each iteration by

$$
\bar{\sigma}^{(l)}=\frac{1}{W}\left(\sum_{i \in D} \frac{z_{i}-1}{\alpha_{i}}+\sum_{j \in \bar{D}} \frac{g\left(\alpha_{j} \bar{\sigma}^{(l-1)}\right)-1}{\alpha_{j}}\right),
$$

where $W$ denotes the length of the sliding window and it is the sum of the number of detections $(N)$, and the number of misses $(M)$ in the window. Note that the function (14) corresponds to an average of the estimates of $\bar{\sigma}$ that are obtained based on the observations of the detections and misses in the window. To be more specific, $\left(z_{i}-1\right) / \alpha_{i}$ in the first term of (14) is an (one-sample) unbiased estimate of $\bar{\sigma}$ with the observed signal strength $z_{i}$ of the detection at scan $i$, and $\left(g\left(\alpha_{j} \bar{\sigma}^{(l-1)}\right)-1\right) / \alpha_{j}$ in the second term is also an one-sample estimate of $\bar{\sigma}$ with the unobserved but estimated signal strength $g\left(\alpha_{j} \bar{\sigma}^{(l-1)}\right)$ of the missed detection at scan $j$. Recall that the unobserved signal strength is estimated by (13) which evaluates the expected value of the undetected signal strength distributed with the pdf of (7) with $\bar{\sigma}=\bar{\sigma}^{(l-1)}$.

It can be shown that the iteration with the mapping (14) forms an instance of a GEM algorithm [9] in the following cases: where $\alpha_{k}$ 's are a constant over a sliding window with scans appearing in (14), and where $\alpha_{k} \bar{\sigma}^{(l)}$ 's and $\alpha_{k} \bar{\sigma}^{(l-1)}$ 's are sufficiently large. In these cases, the sequence $\left\{\bar{\sigma}^{(l)}\right\}$ of (14) satisfies the sufficient conditions (in Theorem 2 of [9]) for convergence to a ML estimate. In the event that the mapping (14) fails to satisfy the inequality $Q\left(\bar{\sigma}^{(l)}, \bar{\sigma}^{(l-1)}\right) \geq Q\left(\bar{\sigma}^{(l-1)}, \bar{\sigma}^{(l-1)}\right)$, a possible simplest step is
TABLE 1: RMS estimation errors for $\bar{\sigma}=1$.

\begin{tabular}{lcccc}
\hline & \multicolumn{4}{c}{$\overline{\mathrm{SNR}}\left(P_{D}\right)$} \\
& $8(0.46)$ & $16(0.67)$ & $32(0.81)$ & $64(0.90)$ \\
\hline $\mathrm{ML}(N=5)$ & 0.362 & 0.392 & 0.417 & 0.426 \\
$\operatorname{ML}(N=10)$ & 0.252 & 0.278 & 0.290 & 0.307 \\
$\operatorname{ML}(W=7)$ & 0.439 & 0.405 & 0.384 & 0.387 \\
$\operatorname{ML}(W=10)$ & 0.360 & 0.337 & 0.326 & 0.317 \\
$\operatorname{ML}(W=12)$ & 0.332 & 0.311 & 0.297 & 0.295 \\
$\operatorname{ML}(W=15)$ & 0.298 & 0.273 & 0.266 & 0.263 \\
$\operatorname{MED}$ & - & 0.331 & 0.340 & 0.364 \\
\hline
\end{tabular}

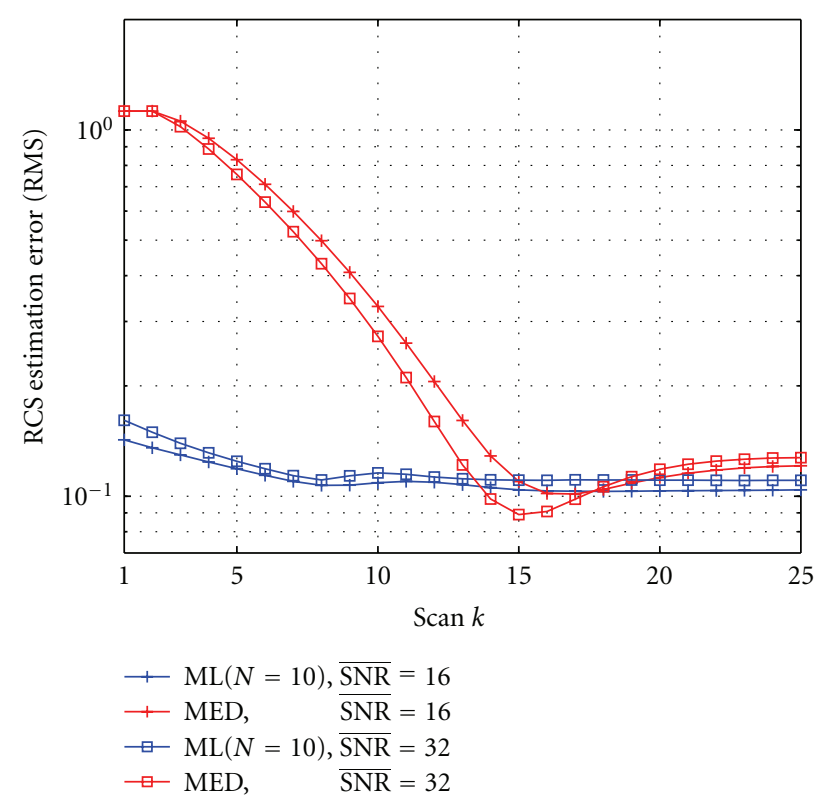

FIGURE 1: RMS estimation errors for $\bar{\sigma}=0.375$.

to stop the iteration and return $\bar{\sigma}^{(l-1)}$ as an estimate. The GEM algorithm was implemented to compute a ML estimate, and its estimation performance is discussed in the following section.

\section{Numerical Experiments}

We performed numerical experiments to investigate RCS estimation performance of the ML approach. The ML estimates were evaluated with iterations of (14) that form an implementation of the GEM algorithm. The algorithmic procedure MED was also implemented and its performance was compared with that of the ML estimation. The procedure estimates the average RCS using the median difference between SNR measurements of return signals in a sliding window and their corresponding expected values. The procedure uses $z_{k}-1$ as the measurement of SNR at scan $k$ [3] and a sliding window with 5 detections. The experiments were performed for $P_{F}=10^{-3}$.

Firstly, we obtained the root-mean-squared (RMS) estimation errors of the average RCS $(\bar{\sigma})$, when the true value 


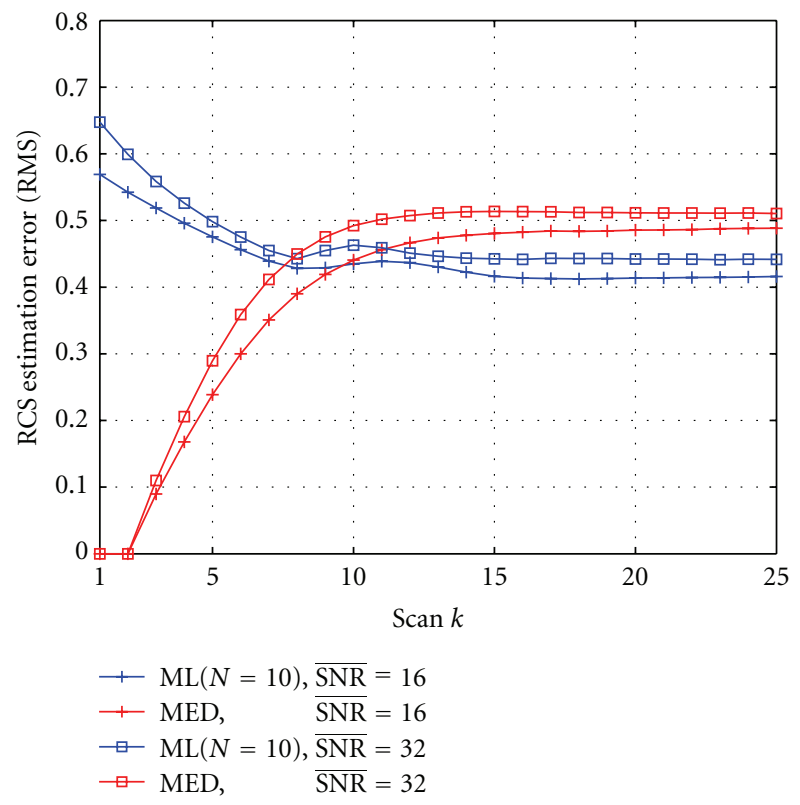

FIGURE 2: RMS estimation errors for $\bar{\sigma}=1.5$.

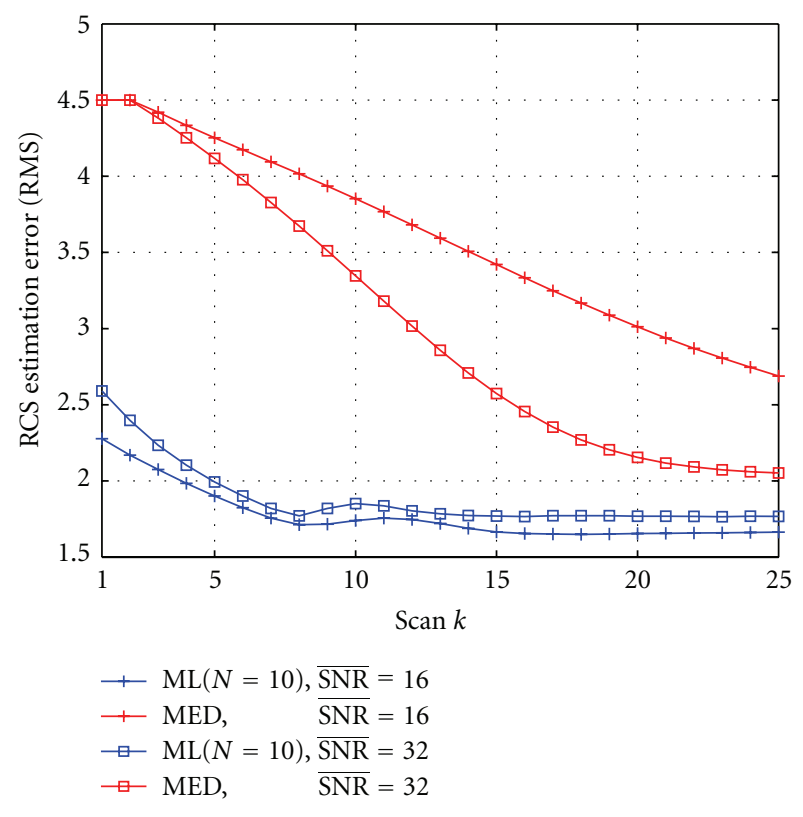

FIGURE 3: RMS estimation errors for $\bar{\sigma}=6$.

is unity. The results are presented in Table 1. The errors of ML estimation were evaluated for a sliding window with a fixed size and for a sliding window with a fixed number of detections in it. In the table, $\operatorname{ML}(N=n)$ denotes ML estimation using a sliding window with $n$ detections, and $\operatorname{ML}(W=w)$ denotes ML estimation using a sliding window with a fixed length $w$. Note that the window size of $\operatorname{ML}(W=$ $w$ ) is fixed to $w$ regardless of the number of detections in the window. On the contrary, the window size of $\operatorname{ML}(N=n)$ can change to retain $n$ detections in the window. The average
SNR, $\overline{\mathrm{SNR}}$, was set to a constant having a value of $8,16,32$, and 64. Note that each of the RMS errors in the table was obtained based on $10^{5}$ estimates evaluated along a random sequence of detections and misses. The random sequence was generated according to the signal and detection model described in Section 2. The iteration of (14) stops when the step size becomes less than $10^{-3}$.

The table shows that the RMS errors of $\operatorname{ML}(N=5)$ and $\operatorname{ML}(N=10)$ decrease with $\overline{\mathrm{SNR}}$. It appears counterintuitive, but the decrease is due to more information on $\bar{\sigma}$ gathered over a sliding window whose length increases in average as $\overline{\mathrm{SNR}}$ decreases. Note that as $\overline{\mathrm{SNR}}$ decreases, the probability of detection decreases, and more scans are required in average to retain a specified number of detections. In contrast, the RMS error of $\operatorname{ML}(W=w)$ decreases as $\overline{\mathrm{SNR}}$ increases. This implies that detections are more informative than misses in estimating $\bar{\sigma}$, since they provide additional information on $\bar{\sigma}$ via signal strength observations. In all cases, the error decreases as the window size increases through $W$ or $N$. The window size of $\operatorname{ML}(N=n)$ corresponds to the size of $\operatorname{ML}(W=w)$ in effect with $w=n / P_{D}$. For instance, $N=10$ corresponds approximately to $W=15$ for $\overline{\mathrm{SNR}}=16\left(P_{D}=\right.$ $0.67)$ and $W=12$ for $\overline{\mathrm{SNR}}=32\left(P_{D}=0.81\right)$. Table 1 shows that the errors of $\operatorname{ML}(N=10)$ and $\operatorname{ML}(W=15)$ are 0.278 and 0.273 for $\overline{\mathrm{SNR}}=16$, respectively, and those of $\operatorname{ML}(N=10)$ and $\operatorname{ML}(W=12)$ are 0.290 and 0.297 for $\overline{\mathrm{SNR}}=32$, respectively. The numbers are comparable to each other, and it implies that $\mathrm{ML}(N)$ does not have any particular advantages over $\operatorname{ML}(W)$ in practice.

The table also shows that MED outperforms $\operatorname{ML}(N=5)$ and $\operatorname{ML}(W=7)$ for higher signal strength, but it is less effective than $\operatorname{ML}(N=10)$ and $\operatorname{ML}(W=10)$. It should be remarked that MED failed to estimate $\bar{\sigma}$ for $\overline{\mathrm{SNR}}=8$ (the estimates of MED were close to zero). Note that MED uses a sliding window with 5 detections. We also performed numerical experiments to obtain the RMS errors of MED for a sliding window with 10 detections. It failed again to estimate $\bar{\sigma}$ for $\overline{\mathrm{SNR}}=8$, and all the errors for higher $\overline{\mathrm{SNR}}$ 's were larger than those of MED with 5 detections. It appears that MED was designed based on 5 detections and it needs a modification for a different number of detections to assure its best performance. The experimental results indicate that ML estimation can perform successfully for a low SNR target that MED fails to estimate. The computational cost of ML estimation was not significant. The GEM terminated in 3.59 iterations of (14) in average and in maximum 5 iterations to yield a ML estimate.

Additional experiments were performed to investigate estimation accuracy at the early stage of tracking. The ensemble averaged RMS errors are presented in Figures 1 to 4 for the true value of $\bar{\sigma}$ with $0.375,1.5$, and 6 . The average was evaluated over $10^{5}$ time sequences of estimates. The track was initiated according to the " 3 out of 5 " logic [16], and the estimation is activated at the third detection of the initiation logic. The scan index $k$ was set to one at the start, and the initial value of the RCS estimate was set to 1.5 . The window size of $\operatorname{ML}(N=10)$ was adjusted to continue to increase until the window holds 10 detections and to maintain 10 detections at subsequent scans. Figures 1 


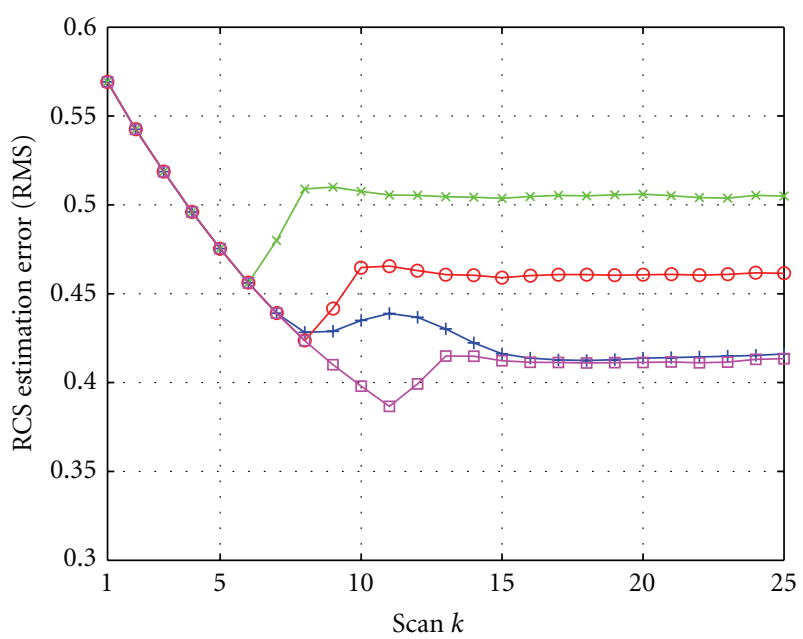

(a) $\overline{\mathrm{SNR}}=16$

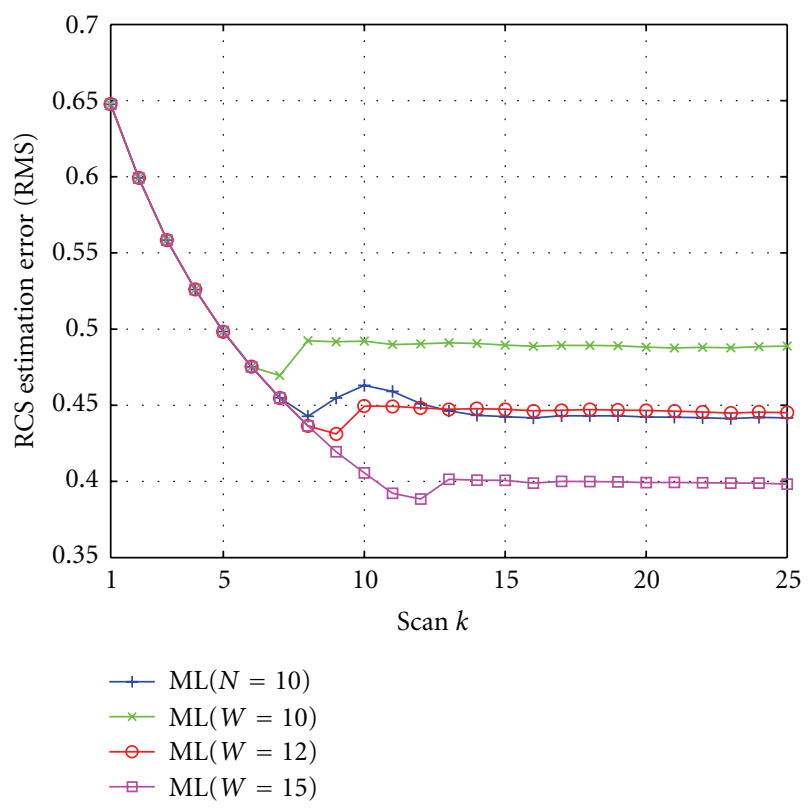

(b) $\overline{\mathrm{SNR}}=32$

FIgURE 4: RMS estimation errors for $\bar{\sigma}=1.5$.

and 3 show that the ML estimation is much more accurate than MED at the first 10 scans. Figure 2 presents the RMS errors for the case that the initial estimate of MED is perfect. In this case, the error of MED is zero at the first and second scans. Note that the ML estimation, however, is not susceptible to the accuracy of a preset initial value of the RCS estimate.

Figures 1 to 3 show that the errors for $\overline{\mathrm{SNR}}=16$ each decrease eventually to the values slightly lower than those for $\overline{\mathrm{SNR}}=32$. This observation is consistent with the results presented in Table 1. Note, furthermore, that $\mathrm{ML}(N=10)$ maintains the error for $\overline{\mathrm{SNR}}=16$ to be smaller than that for $\overline{\mathrm{SNR}}=32$ at all scans. This confirms that the argument on the results of the table holds for ML $(N=10)$ over all scans, including the early stage of tracking. In contrast, MED decreases its error faster at the early stage for $\overline{\mathrm{SNR}}=32$ than for $\overline{\mathrm{SNR}}=16$; see Figures 1 and 3 . This is the case where the initial estimate error is larger than the "steadystate" error. In this case, MED is activated and starts to correct the estimation error at an earlier scan for $\overline{\mathrm{SNR}}=32$ since the window can retain 5 detections faster when the probability of detection is higher. This correction allows to pass a more accurate initial estimate to the MED estimation of the following scan and causes the estimation error to decrease faster. Conversely, MED starts to miscorrect the error at an earlier scan for $\overline{\mathrm{SNR}}=32$ in Figure 2, where the initial estimate error is smaller than the "steady-state" error. This miscorrection passes a worse initial estimate to the MED estimation of the next scan. This affects adversely the estimation and causes the error to increase faster.

Figure 4 presents the RMS errors of $\operatorname{ML}(N=10)$ and $\operatorname{ML}(W=w), w=10,12$, and 15 , for $\bar{\sigma}=1.5$. At the early scans, $\operatorname{ML}(N=10)$ and $\operatorname{ML}(W=w)$ use the same windows and the RMS errors coincide as a consequence. The "steady-state" errors are also consistent with the results presented in Table 1. The transient "dynamics" of $\operatorname{ML}(W)$ can be described as follows. $\mathrm{ML}(W)$ starts at scan 1 with three detections as it is activated according to the " 3 out of 5 " track initiation logic. The three detections according to the logic requires 3.9 and 3.6 scans in average for $\overline{\mathrm{SNR}}=16$ and 32 , respectively. This implies that $\operatorname{ML}(W=w)$ requires $w-3.9$ and $w-3.6$ more scans in average for $\overline{\mathrm{SNR}}=16$ and 32 , respectively, to stop expanding its window. Suppose that, for instance, $w=12$. In this case, the stopping starts to occur at scan 8 and occurs in average between scans 9 and 10. Note that the statistical characteristics of the observations before and after $k=1$ are different due to the track initiation logic, since the logic intervenes in effect to select observations with a higher probability to retain more detections in the window at scans earlier than and at scan 1 . The detections are more informative than misses in the RCS estimation. It is shown in Figures 4(a) and 4(b), respectively, that $\operatorname{ML}(W=12)$ begins to lose the better quality information by releasing the detections from the window and its errors start to increase at scan 8 and at scan 9. This explains the reason that the "undershoot" occurs at scan 8 and scan 9, respectively, for $\overline{\mathrm{SNR}}=16$ and 32 .

The transient "dynamics" of $\operatorname{ML}(N=10)$ in Figures 1 to 4 can be explained based on the arguments similar to the case of $\operatorname{ML}(W=12) \cdot \operatorname{ML}(N=10)$ starts at scan 1 with three detections according to the " 3 out of 5 " logic. It increases its window size until the window retains 7 more detections, which requires 10.5 and 8.6 additional scans in average for $\overline{\mathrm{SNR}}=16$ and 32, respectively. This implies that $\operatorname{ML}(N=10)$ begins to stop expanding its window at scan 8 and stops in average around at scan 11 for $\overline{\mathrm{SNR}}=16$ and at scan 9 for $\overline{\mathrm{SNR}}=32$. As discussed earlier, the stopping causes to lose the quality information and increase the estimation errors. This explains why the error starts to increase at scan 8 and scan 9, and it increases until scan 11 and scan 10 to generate the "overshoot" for $\overline{\mathrm{SNR}}=16$ and 32, respectively. The transient "dynamics" of MED seems more complicated than in the case of $\operatorname{ML}(N=10)$. Its analysis is not pursued in this paper. 


\section{Conclusion}

An ML approach has been presented for estimating the average RCS, and a numerical solution to the approach has been proposed based on a generalized expectation maximization algorithm. Numerical experiments were performed to compare the RCS estimation performance of the ML approach with that of a previously reported procedure MED. The experimental results show that the ML approach can perform successfully even for a low-SNR target that MED fails to estimate. The results also show that, in contrast to MED, the ML approach is not susceptible to the error of a preset initial value of the RCS estimate at the early stage of tracking. Extension to the case in the presence of false alarms is currently under investigation.

\section{Acknowledgment}

This work was supported by the BK-21 Program.

\section{References}

[1] S. S. Blackman and R. Popoli, Design and Analysis of Modern Tracking Systems, Artech House, Norwood, Mass, USA, 1999.

[2] R. F. Popoli, S. S. Blackman, and M. T. Busch, "Application of multiple-hypothesis tracking to agile beam radar tracking," in Signal and Data Processing of Small Targets, vol. 2759 of Proceedings of SPIE, pp. 418-428, Orlando, Fla, USA, April 1996.

[3] G. van Keuk and S. S. Blackman, "On phased-array radar tracking and parameter control," IEEE Transactions on Aerospace and Electronic Systems, vol. 29, no. 1, pp. 186-194, 1993.

[4] T. Kirubarajan, Y. Bar-Shalom, W. D. Blair, and G. A. Watson, "IMMPDAF for radar management and tracking benchmark with ECM," IEEE Transactions on Aerospace and Electronic Systems, vol. 34, no. 4, pp. 1115-1134, 1998.

[5] Y.-H. Jung and S.-M. Hong, "Modeling and parameter optimization of agile beam radar tracking," IEEE Transactions on Aerospace and Electronic Systems, vol. 39, no. 1, pp. 13-33, 2003.

[6] W.-Y. Choi and S.-M. Hong, "Modeling of target detection in local search for phased array radar tracking," International Journal of Control, Automation and Systems, vol. 7, no. 6, pp. 922-929, 2009.

[7] N. Levanon, Radar Principles, Wiley, New York, NY, USA, 1988.

[8] W.-D. Wirth, Radar Techniques using Array Antennas, Institution of Engineering and Technology, London, UK, 2001.

[9] A. P. Dempster, N. M. Laird, and D. B. Rubin, "Maximum likelihood from incomplete data via the EM algorithm," Journal of Royal Statistical Society, Series B, vol. 39, no. 1, pp. 1-38, 1977.

[10] R. L. Streit and T. E. Luginbuhl, "A probabilistic multihypothesis tracking algorithm without enumeration and pruning," in Proceedings of the 6th Joint Service Data Fusion Symposium, pp. 1015-1024, Laurel, Md, USA, June 1993.

[11] R. L. Streit and T. E. Luginbuhl, "Probabilistic multihypothesis tracking," Tech. Rep. NUWC-NPT/10/428, Naval Undersea Warfare Center Division, Newport, RI, USA, February 1995.
[12] H. Gauvrit, J. P. Le Cadre, and C. Jauffret, "A formulation of multitarget tracking as an incomplete data problem," IEEE Transactions on Aerospace and Electronic Systems, vol. 33, no. 4, pp. 1242-1257, 1997.

[13] M. A. Zaveri, S. N. Merchant, and U. B. Desai, "A combined PMHT and IMM approach to multiple-point target tracking in infrared image sequence," EURASIP Journal on Image and Video Processing, vol. 2007, Article ID 19139, 14 pages, 2007.

[14] M. Wieneke and W. Koch, "On sequential track extraction within the PMHT framework," EURASIP Journal on Advances in Signal Processing, vol. 2008, Article ID 276914, 13 pages, 2008.

[15] R. Deming, J. Schindler, and L. Perlovsky, "Multi-target/multisensor tracking using only range and doppler measurements," IEEE Transactions on Aerospace and Electronic Systems, vol. 45, no. 2, pp. 593-611, 2009.

[16] A. Farina and F. A. Studer, Radar Data Processing, vol. 1, Wiley, New York, NY, USA, 1985. 